\title{
Content Based Vector Image Retrieval using LBG Algorithm
}

\author{
Rachana D. Shinde ${ }^{1}$, Lima Mandal ${ }^{1}$, Apeksha Kulkarni ${ }^{1}$, Dr. A. C. Lomte ${ }^{4}$ \\ Department of Computer Engineering, BSIOTR, JSPM, Pune, Maharashtra, India ${ }^{1,2,3}$ \\ BSIOTR, JSPM, Wagholi, Pune, India ${ }^{4}$
}

\begin{abstract}
There are many image retrieval and image compression techniques. Most of the image retrieval techniques are not capable to fastest relevant image retrieval. CBVIR is also known as Content based vector image retrieval or Query based image content. In CBIR, user gives image as an input to the system and after processing system returns similar images of Output. In this work, we present CBVIR using VQ. VQ is a classical quantization technique. VQ captures the spatial relationship between pixel. VQ contains codebook generation, encoding and decoding. Effective image indexing and retrieval technique are essential using codebook. Using CBVIR, retrieval performance is faster than the color based image retrieval. In this paper, we present a snapshot of the recent developed schemes. The discussed schemes include LBG, Enhance LBG (ELBG).
\end{abstract}

Keywords: CBVIR, Vector Quantization (VQ), LBG, ELBG, Image Indexing, Image Retrieval, Image Compression.

\section{INTRODUCTION}

Nowadays people use mobile and web applications like Facebook, Whatsapp, Youtube, We Chat etc. So different kind of images are stored in digital image libraries. To access digital image, retrieval of image has evolved. Formal image retrieving based on attributes of images such as image number, image discription [2] retrieving the similar images. But it have some drawbacks:- firstly limited number of query types and secondly simple attributes cannot give image completely. To overcome this drawback CBVIR technique was born in 90's used. CBVIR also query based image content and Content based image retrieval. Using 2 technique we can retrieve the images. First, Annotational based image retrieval and second Content based Image Retrieval. Annotational is nothing but text based image retrieval. The user gives text as input and then after processing system returns images relevant to text as output. But it also have limitations. For example user wants images of jaguar. He gives input jaguar to the system but the system become confused whether the jaguar is car, logo or animal. To overcome this limitations, Content based vector image retrieval is used.

Here we are used query by example model [1], where user gives image as a input and system returns similar images as output. In CBVIR, each image that is stored in database that has its feature is extracted and compared with features of query images. CBVIR system has 2 steps: first is Feature Extraction which extracts low level features of images. Second is matching extracted features with features of database images.

CBVIR technique also uses low level features like color, shape, and texture that automatically extracted from image[2-3].

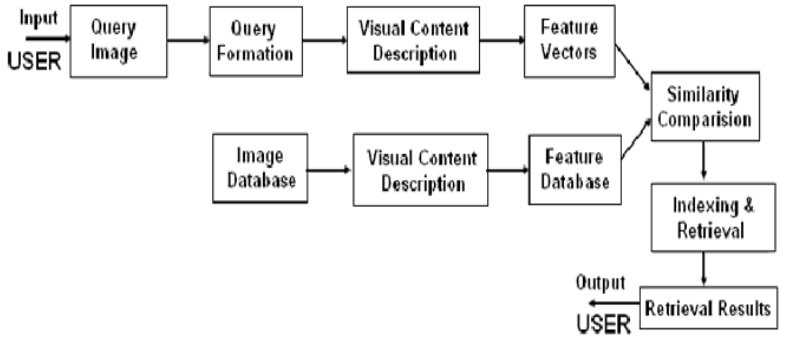

Fig 1. Flow of CBVIR Process

For example in a color based CBVIR system, a histogram of pixels values is used as a parameter for matching and retrieval. But these systems still have many limitations such as it cannot capture spatial relationship among pixels. But till date such systems are still famous. Based on this discussion it is clear that a color based CBVIR systems is required, which can also capture spatial relationship among pixels.

For example in a color based CBVIR system, a histogram of pixels values is used as a parameter for matching and retrieval. But these systems still have many limitations such as it cannot capture spatial relationship among pixels. But till date such systems are still famous. Based on this discussion it is clear that a color based CBVIR systems is required, which can also capture spatial relationship among pixels.

\section{VECTOR QUANTIZATION}

It is a quantization technique based on the principle of block coding in signal processing allows the modeling of probability density functions distribution of prototype vectors. used for data compression and image compression 
it works by dividing a large set of points (vectors) into called the set of all blue lines which result in the smallest groups integration. the same number of points closest to average bend. Assume that there is a training sequence them. Each group is represented by its centroid point using some clustering algorithms.

\section{A. How vector quantization work?}

Vector quantization (VQ) is nothing more than an approximator. The idea is similar to the nearest integer. An example of a 1-dimensional Vector quantization is shown below:

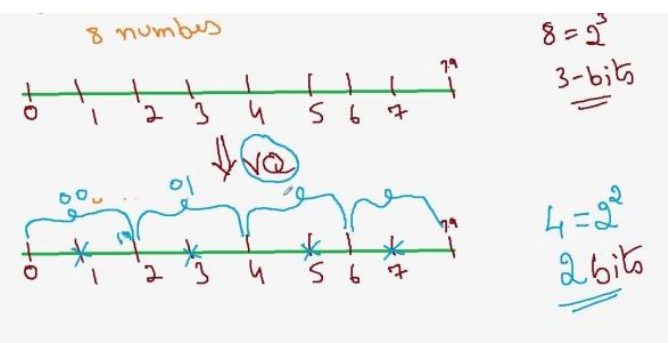

Fig. 2 data compression

Here, Every number between 0 to1.9 are precise by 1.therefore every number less than 2 are precise by 1 . Every number between 2 to 3.9 are approximated by 3 . The precise values are uniquely represented by 2 bits/dimension.

An example of a 2-dimensional Vector quantization is shown below:

In a 2-dimensional Vector quantization, every pair of numbers in a particular region are approximated by a red dots associated with that region. there are 32 regions and 32 red dots each of which can be adversely represented by 4 bits. Thus, this is a 2-dimensional, 4-bit Vector quantization.

In the beyond example, the red dots are called code vectors and the regions assign by the blue borders are called encoding regions. The collection of all code vectors is called the codebook and the collection of all encoding regions is called the partition of the space.

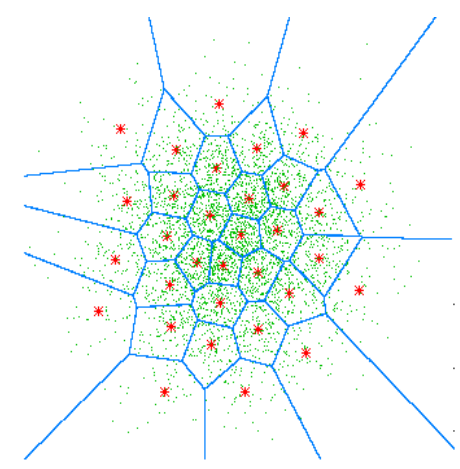

Fig 3 data compression

B. Design Problem

Design problem can be stated in Vector quantization as follows. Vector source with its algebraic acreage called as a distortion measure, and the number of code vectors, find a codebook called the set of all red dots and a partition $\stackrel{\text { which consisting } \quad \text { of }}{\mathcal{T}=\left\{\mathbf{x}_{1}, \mathbf{x}_{2}, \ldots, \mathbf{x}_{M}\right\}}$

source vectors:

This training sequence can be accessed from some large database. For example, if the antecedent is a speech signal, then we can say that training sequence can be accessed by recording a few long telephone conversations. ${ }^{M}$ is accept to be abundantly large so that all the algebraic properties of the antecedent are acquired by the training sequence. accept that the source vectors are $k$ dimensional, example,

$$
\mathbf{x}_{m}=\left(x_{m, 1}, x_{m, 2}, \ldots, x_{m, k}\right), \quad m=1,2, \ldots, M .
$$

$N$

consider be the number of code vectors and consider $\mathcal{C}=\left\{\mathbf{c}_{1}, \mathbf{c}_{2}, \ldots, \mathbf{c}_{N}\right\}$,

Act as the codebook. Each code vector is ${ }^{k}$-dimensional, example.,

$\mathbf{c}_{n}=\left(c_{n, 1}, c_{n, 2}, \ldots, c_{n, k}\right), \quad n=1,2, \ldots, N$.

assume ${ }^{S_{n}}$ be the encoding region accomplice with code vector $\mathbf{c}_{\boldsymbol{n}}$ and assume

$\mathcal{P}=\left\{S_{1}, S_{2}, \ldots, S_{N}\right\}$

denote the partition of the space. If the source vector $\mathbf{x}_{m}$ is in the encoding region $S_{n}$, then its approximation (denoted by $Q\left(\mathbf{x}_{m}\right)$ ) is $\mathbf{c}_{n}$ :

$Q\left(\mathbf{x}_{m}\right)=\mathbf{c}_{n}, \quad$ if $\mathbf{x}_{m} \in S_{n}$.

let a squared-error distortion measure, the average distortion is given by:

$$
\begin{gathered}
D_{\text {ave }}=\frac{1}{M k} \sum_{m=1}^{M}\left\|\mathbf{x}_{m}-Q\left(\mathbf{x}_{m}\right)\right\|^{2}, \\
\|\mathbf{e}\|^{2}=e_{1}^{2}+e_{2}^{2}+\ldots+e_{k}^{2}
\end{gathered}
$$

where

The design $\mathcal{T}$ and ${ }_{N}^{\text {problem can be succinctly stated as follows: Given }} \mathcal{\mathcal { C }} \underset{\text { and }}{\mathcal{P}} D_{\text {ave }}$ is minimized

\section{Optimality Criteria}

${ }_{\text {If }}^{\mathcal{C}}$ and $\mathcal{P}$ are a solution to the above minimization problem, then it must satisfy the following two criteria.

a) Nearest Neighbor Condition:

$$
S_{n}=\left\{\mathbf{x}:\left\|\mathbf{x}-\mathbf{c}_{n}\right\|^{2} \leq\left\|\mathbf{x}-\mathbf{c}_{n^{\prime}}\right\|^{2} \forall n^{\prime}=1,2, \ldots, N\right\}
$$

This condition says that the encoding region $S_{n}$ should consist of all vectors that are closer to $\mathbf{c}_{n}$ than any of the 
Vol. 6, Issue 1, January 2017

other code vectors. For those vectors lying on the boundary (blue lines), any tie-breaking procedure will do.

b) Centroid Condition:

$$
\mathbf{c}_{n}=\frac{\sum_{\mathbf{x}_{m} \in S_{n}} \mathbf{x}_{m}}{\sum_{\mathbf{x}_{m} \in S_{n}} 1} \quad n=1,2, \ldots, N
$$

This condition says that the code vector $\mathbf{c}_{n}$ should be average of all those training vectors that are in encoding region ${ }^{S_{n}}$. In an implementation, one should ensure that at least one training vector belongs to each encoding region (so that the denominator in the above equation is never 0).

Vector Quantization consist of three procedures, which are Codebook Generation Process, Encoding procedure and Decoding procedure. Codebook generation process is done using LBG algorithm. In 1980, Linde, Buzo, and Gray advised a vector quantization design algorithm established on a training sequence. The use of a training sequence bypasses the need for multi-dimensional integration for an image compression in an adequate way. The main equitable of this study is to generate and compare the codebook for a vector quantizer using Linde Buzo algorithm $[2,3]$.

D. Vector Quantization Encoding and Decoding Process In data Encoding and Decoding process Encoding is the process of converting plain data into something that appears to be random and meaningless called as complex data. Decoding is the opposite process it converting complex data back to plain data [3].

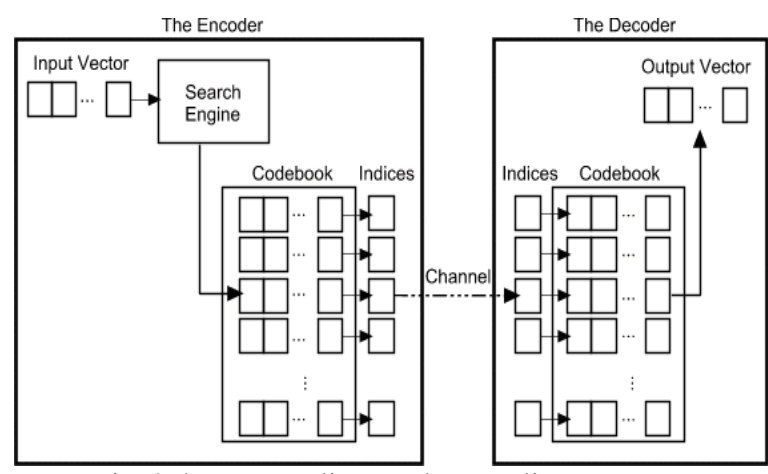

Fig.4 data Encoding and Decoding process

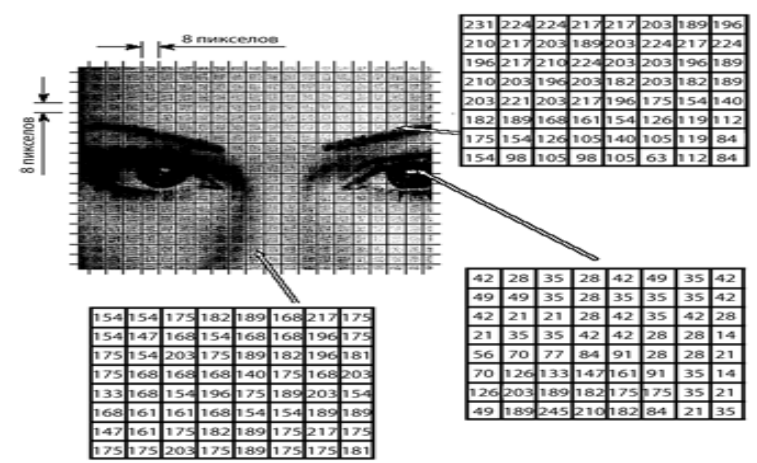

Fig.4 JPEG image division jpeg transform compression start by breaking the image into $8 * 8$ groups, each containing 64 pixels.three of these $8 * 8$ groups are enlarge in this figure, showing the values of the individual pixels, a single byte value between 0to 255 .

\section{E. Euclidean Distance}

In mathematics, the Euclidean metric or Euclidean distance is the straight-line distance between two points in Euclidean space. Using this distance, Euclidean space becomes a metric space. In the Euclidean distance, the associated norm is called the Euclidean norm. Older one literature refers to the metric as Pythagorean metric.

$$
\text { Euclidean Distance }=d=\sqrt{\sum_{i=1}^{N}(X i-Y i)^{2}}
$$

\section{LBG ALGORITHM}

A zero tree consists of variable block sizes of the similar information that belongs to different layers. The proposed encoder produces the codebooks and codewords of each subband using LBG algorithm.

LBG algorithm steps:

Step 1: set $M=1$ ( $M$ is a number of partition or cell) find the centroid of all the training data.

Step 2: split $\mathrm{M}$ into the $2 \mathrm{M}$ partition by splitting each current codeword by finding two points that are far apart in each partition using a heuristic method, and use these two points as the new centroid for the new $2 \mathrm{M}$ codebook now set $M=2 M$.

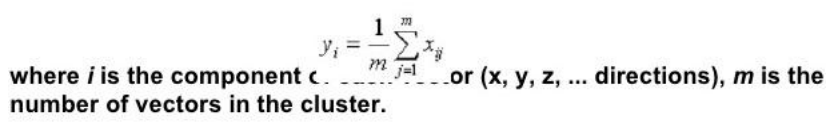

Step 3: now use an iterative algorithm to reach the best set of the centroid for new codebook.

Step 4: if M equal the VQ codebook size require, STOP otherwise ho to step 2.

\section{STRUCTURE OF CODEBOOK}

The goal of VQ image compression, instead of loosing image quality achieve compression high ratio. Actually meaning of this capture all the low level features and stored generated codewords in small space as possible. All this happens using colors pace. Suppose codebook is generated using RGB colorspace. Every codebook is represented by code words. In short codebook is a storage of codewords which is nothing but secret word or encrypted word. Every codebook represents channel of colors. Image Retrieval based on Vector Quantization. Here two conditions must be true for image retrieval:

1. Images which are similar have same histogram.

2. Sometimes images with same histogram are perceptually similar but actually not.

After codebook generation encoding process is done on query image. As a result 3 indices are represents each image vector where each index represents the best match 
Vol. 6, Issue 1, January 2017

codeword with respective to color [1,2] channel. After indexing histogram for each color is generated which is represented for RGB colorspace as $\mathrm{Hr}, \mathrm{Hg}$, Hb. Histogram is generated for any colorspace. When comparing histogram is done we could think that two images are similar. However, they look perceptually different by estimating color channels in two images group together in differently.

To comparison of similarity between two images, instead of three codebook only one codebook is generated. So here only one histogram is generated and used. Each bin in histogram is used to count occurences of each codeword. Here perceptual similarity between images is calculated from similarity of Histogram. we need more codebook storage than traditional technique. This storage is 4096 times greater than traditional one. This codebook has enough storage space which takes atleast the features of images. The similar images are retrieved, as long as database image have similar or exact feature of query image. Retrieved Images can be ranked in an ascending order of distance calculated. The smaller the calculated distance between two images, the images are similar or larger the distance between two images, more different two images.

\section{RESULTS AND DISCUSSION}

In our project user gives image as input. After that Vector quantization technique is applied on that input. At the end result of similar images will be retrieved from image database. All this is done on the basis of calculation of the distance using standard formula (i.e. Euclidean Distance) and Similarity Matching.

\section{CONCLUSION}

In this paper we have proposed a new technique for CBVIR system which is mainly based on 2: Image Compression and Image Indexing. We discuss the methods of vector quantization that is codebook generation, encoding and decoding. Image retrieval easy because of Codebook. Our Future research will be overall progress of each quality point of image retrieval using compressed database using another technique. Using this project we can develop any type of search engine for Forensic.

\section{ACKNOWLEDGMENT}

The Authors also gratefully acknowledge the helpful suggestions and conversation with Virendra Ravaji, which have improved the knowledge.

\section{REFERENCES}

[1] Ajay H. Daptardar and James A. Storer Computer, "ContentBased Image Retrieval via vector Quantization, Science Department, Brandeis University, Waltham, MA 02454, USA
[2] ShyhWei Teng*, Guojun Lu, "Image indexing and retrieval based on vector quantization" Gippsland School of Information Technology, Monash University, Gippsland Campus, Churchill, Vic. 3842, Australia

[3] Tzu-Chuen Lu "A Survey of VQ Codebook Generation" Department of Information Management Chaoyang University of Technology Taichung,Taiwan, Ching-Yun Chang Computer Laboratory University of Cambridge Trinity Lane, Cambridge CB2 1TN,UK Received March 2010; revised April 2010

[4] K Idris, F., Panchanathan, S.: Image and Video Indexing using Vector Quantization. Machine Vision and Applications 10 (1997) 43-50

[5] Idris, F., Panchanathan, S.: Storage and Retrieval of Compressed Images. IEEE Transactions on Consumer Electronics 43 (1995) 937-941

[6] Lu, G., Teng, S.: A Novel Image Retrieval Technique based on Vector Quantization. In: Proceedings of International Conference on Computational Intelligence for Modeling, Control and Automation. (1999) 36-41.

[7] Gersho, R.M. Gray, Vector Quantization and Signal Compression,Kluwer Academic Publishers, Dordrecht, 1992.

[8] H. Abut (Ed.), Vector Quantization, IEEE Press, New York, 1990.

[9] K. Sayood, Introduction to Data Compression, second ed.,Morgan Kaufmann Publishers Inc., San Francisco, CA, 2000.

[10] Schaefer, G.: Compressed Domain Image Retrieval by Comparing Vector Quantization Codebooks. In: Proceedings ofthe SPIE Visual Communications and Image Processing. Volume 4671. (2002) 959966.

[11] W. Niblack, et al., QBIC Project: querying images by content, using color, texture, and shape, Proceedings of Conference on Storage and Retrieval for Image and Video Databases, 1-3 February 1993, San Jose, CA, US, SPIE, vol. 1908, 1993, pp. 1908-1920.

[12] M.J. Swain, D.H. Ballard, Color indexing, Int. J. Comput. Vision 7 (1991) 11-32

[13] G. Lu, Image retrieval based on color, Proceedings of the Conference on Storage and Retrieval for Image and Video Databases IV, San Jose, CA, 1- 2 February 1996, SPIE Proceedings Series, vol. 2670. 